\title{
Towards fivefold symmetry?
}

\author{
Crystallography is in for a minor upheaval, with the recognition of forbidden \\ icosahedral symmetry by both construction and experiment.
}

THAT crystals never have fivefold axes of symmetry has been part of the doctrine of crystallography since 1895, when Fedorov first classified the admissible symmetry groups. The reasons are straightforward. Consecutive rotations of an infinite crystal lattice in either sense about one or more symmetry axes must yield an identical structure, identically placed in space. But rotations by $2 \pi / 5$ in opposite directions about neighbouring fivefold axes would break this rule.

Grandly, it might be said that the inadmissibility of fivefold crystal symmetry says something about the character of two and three-dimensional space. Similarly, it is not possible to tile a plane with equilateral polygons or to fill three-dimensional space with icosahedrons, the regular twenty-faced polyhedra most easily described as a pair of opposed pentagonal pyramids fashioned from equilateral triangles and joined together, base to base, by a band of ten opposed identical triangular faces.

Both by accident and design, people have been whittling away at these restrictions for the past decade, ever since the demonstration by $\mathbf{R}$. Penrose (Oxford) that, although it is not possible to tile the two-dimensional plane with regular pentagons, that can be done with a pair of specific rhombi so as to give a pattern with local fivefold symmetry (see figure). The price for this is that the pattern lacks the translational symmetry of a familiar lattice. The argument has been extended to three dimensions by A.L. Mackay (Birkbeck, London), who has shown that space can be packed by acute and obtuse rhombohedra (see, for example, Physica 114A, 609; 1982). Again, translational symmetry is forfeit to the gain of local fivefold rotational symmetry.

The bearing of all this on real crystallography is far from remote. The textbooks are mostly about infinite and perfect crystal lattices, but real crystals may be small and imperfect. Indeed, X-ray diffraction patterns with fivefold symmetry have been reported from gold, and interpreted as a consequence of twinning on the microscopic scale of the unit cell. But fivefold symmetry should also occur if, for example, metals are deposited from the vapour, when icosahedral packing should be energetically favoured. In November last year, Schechtman et al. (Phys. Rev. Lett. 53, 1951 ; 1984) described the recognition by means of electron diffraction of icosahedral symmetry in grains of a 14 per cent alloy of $\mathrm{Mn}$ in $\mathrm{Al}$, arguing for longrange order of some kind.

This mixture of theorizing and observation has now been put on a broad foundation by $D$. Levine and P.J. Steinhardt (Phys. Rev. Lett. 53, 2477; 1984 ) in a summary of largely unpublished theoretical investigations. The objective is to provide a framework for discussing what they call "quasi-crystals".

Again, the starting point is the Penrose tiling of the plane. The outstanding feature of that network is that it lacks translational symmetry, but it has long-range order in that the orientations of the nearestneighbour bonds (ten altogether, in five oppositely directed pairs) are constant throughout the network. This is the prototype of the quasi-crystal. Bond orientation takes over from translational periodicity.

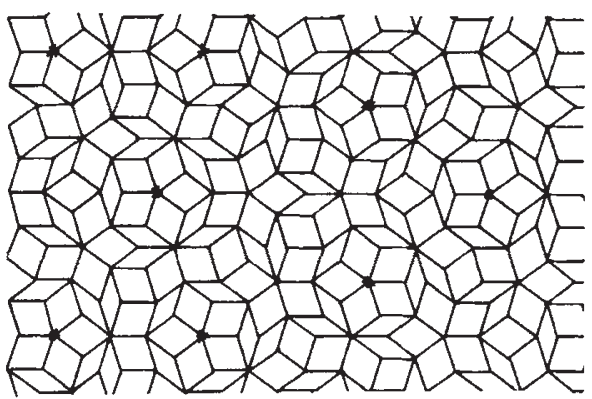

But how to describe a quasi-crystal analytically? Here is the recipe, for a twodimensional net. Define a set of unit vectors $\mathbf{e}_{i}$ oriented along the axes of the underlying polygon, where the index $i$ runs from 1 to $N$, the order of the polygon. Each lattice point can be defined as a linear combination of any two of these vectors, with coefficients $x_{i}$ say, but the requirement that the lattice should be a quasi-crystal is that the sequence of projected lattice distances $x_{i}$ should be the same for all the vectors $\mathbf{e}_{i}$.

This qualification is not a mere nuisance but the essence of the problem. Levine and Steinhardt conclude that the allowable coordinates for quasi-crystals in two or three dimensions are the positions of lattice points in some one-dimensional quasiperiodic sequence, itself constructed by means of a specific set of rules of which the simplest (leading to the Penrose lattice) is the sequence due to Fibonacci, the thirteenth century Italian who made arabic numerals fashionable in Europe.
The rule is simple. Let there be two intervals of length, $r$ and $s$, and construct a sequence iteratively, by replacing $r$ by $r s$ and $s$ by $r$. It is easily seen (by starting with a sequence consisting only of $r$ or $s$ ) that the result is the successive addition of new pieces to what is otherwise a steadily lengthening string of intervals, which may be thought of as a one-dimensional lattice (defining the coordinates $x_{i}$ ). The arrangement is plainly not random; pairs of the intervals $s$ never occur in sequence, for example. But it is also not periodic. Moreover, for a sufficiently long sequence, the intervals $r$ and $s$ occur in the ratio $(1+$ $\sqrt{5} / 2$, the golden mean of antiquity which is also one of the solutions of the equation $\tau^{2}=\tau+1$. If the intervals $r$ and $s$ are also in the ratio of the golden mean, the result is a quasi-periodic sequence that defines the Penrose pattern.

Much of the interest in what Levine and Steinhardt have done is their generalization of this procedure. Their schemes for constructing other quasi-periodic sequences for defining lattice coordinates will provoke a search for other kinds of forbidden symmetry. The applications to three dimensions are described only heligraphically, but Levine and Steinhardt appear to have recovered Mackay's result that icosahedral symmetry in three dimensions can be obtained from a set of interpenetrating rhombic tricontrahedra (shapes with 30 faces, 32 vertices and 60 edges). This analytical account should stimulate both the study of other quasisymmetries and the calculation of their physical properties.

Levine and Steinhardt produce their most startling success in this second connection, with a calculation of the X-ray diffraction pattern from a quasi-periodic icosahedral crystal. That a quasi-crystal should yield diffraction spots is not all that surprising, for the lattice must be built from a small number of recurring intervals. But the big surprise is that Levine and Steinhardt recover almost exactly the pattern found by Schechtman et al.

For crystallographers, the implications are far-reaching. There will be a search for other forbidden symmetry. What the new theory suggests for crystal growth is intriguing; which regular lattices most closely correspond to those of which quasicrystals? What about band-structure in quasi-crystals? Whatever the outcome, crystallographers will have to mug up some unfamiliar mathematics. John Maddox 